Images dans le monde ibérique et ibéricoaméricain

$5 \mid 2012$

Le visible et l'invisible dans le monde hispanique et hispano-américain

\title{
Invisible et indicible dans Cría Cuervos (1975) de Carlos Saura
}

Marianne Bloch-Robin

\section{(2) OpenEdition \\ Journals}

\section{Édition électronique}

URL : http://journals.openedition.org/agedor/909

DOI : 10.4000 /agedor.909

ISSN : 2104-3353

Éditeur

Laboratoire LISAA

Référence électronique

Marianne Bloch-Robin, «Invisible et indicible dans Cría Cuervos (1975) de Carlos Saura », L'Âge d'or [En ligne], 5 | 2012, mis en ligne le 01 mars 2012, consulté le 16 décembre 2019. URL : http://

journals.openedition.org/agedor/909; DOI : 10.4000/agedor.909 


\title{
INVISIBLE ET INDICIBLE DANS CRÍA CUERVOS (1975) DE CARLOS SAURA
}

\begin{abstract}
Résumé : Dans le film Cría Cuervos (1975) de Carlos Saura, la musique qui intervient très peu d'un point de vue quantitatif tient cependant un rôle primordial au sein de la narration cinématographique. Parmi les trois morceaux de répertoire qui constituent l'unique matière musicale du film, une chanson, Porque te vas, composée par José Luis Perales et interprétée par Jeanette, imprègne l'œuvre de ses quatre occurrences. Cette mélodie remplit de multiples fonctions narratives et esthétiques dans son rapport à l'image qui permettent de mettre au jour différentes «strates » invisibles dans le récit filmique. Elle révèle l'intériorité d'Ana, la jeune protagoniste, qui est incapable de formuler ses sentiments refoulés. Par ailleurs, les citations de la chanson créent un parcours narratif particulier au sein de l'œuvre, invisible à première vue, qui permet de mettre en place un récit second constitué par les différentes séquences que «balise» la musique. Enfin, la chanson permet de révéler le point de vue dissimulé de l'énonciateur filmique qui est dévoilé par la toute dernière occurrence extradiégétique.
\end{abstract}

Mots-clés : Carlos Saura - musique vocale - chanson - narration - esthétique - Cría cuervos Porque te vas.

Resumen: En la película Cría Cuervos (1975) de Carlos Saura, la música que interviene muy poco desde un punto de vista cuantitativo representa sin embargo un papel central en la narración cinematográfica. Entre las tres obras de repertorio que constituyen la única música de la película, una canción, Porque te vas, compuesta por José Luis Perales e interpretada por Jeanette, impregna la obra con sus cuatro ocurrencias. Esta melodía cumple múltiples funciones narrativas y estéticas en su relación con la imagen, unas funciones que descubren distintos "estratos" invisibles en el relato fílmico. La canción revela la interioridad de Ana, la joven protagonista, que es incapaz de formular sus sentimientos reprimidos. Por otra parte, las difusiones de la canción crean un recorrido narrativo particular en la obra, invisible a primera vista y que la música desvela. Por fin, Porque te vas permite la revelación del punto de vista disimulado del enunciador fílmico, que se descubre en la última ocurrencia extradiegética.

Palabras clave: Carlos Saura - música vocal - canción - narración - estética - Cría cuervos - Porque te vas. 


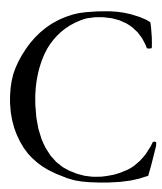

ría cuervos constitue, à bien des égards, un film charnière dans l'œuvre de Carlos Saura. Dernier film tourné sous le franquisme en 1975, il s'agit en quelque sorte de l'aboutissement d'une première étape de la filmographie du réalisateur aragonais qui a reflété, de façon détournée en raison de l'implacable censure de la dictature, le joug que ce régime imposait à la société espagnole. D'un point de vue musical, ce long-métrage ouvre paradoxalement une nouvelle étape, marquée par le bannissement de la musique originale et de ses exploitations traditionnelles ${ }^{1}$ et par l'utilisation exclusive de compositions préexistantes. Trois morceaux de répertoire constituent à eux seuls toute la matière musicale du film : deux pièces vocales - les chansons Porque te vas de José Luis Perales et ;Ay Maricruæ! de Salvador Valverde, Rafael de León et Manuel López Quiroga ${ }^{2}$ et une pièce pour piano Canción y danza ${ }^{\circ} 5$ du compositeur catalan Federico Mompou. Si la musique n'intervient que pendant $20 \%$ de la durée totale du film, ce qui est assez peu d'un point de vue quantitatif, les diffusions réitérées des différents morceaux et la mise en avant du canal sonore imprègnent toute la matière filmique.

L'univers de l'enfance, déjà fondamental, mais néanmoins envisagé depuis l'âge adulte dans le film précédent de Saura, La prima Angélica, est placé au cœur de Cría cuervos. En effet, si ce film présente une triple structure temporelle - le récit de l'enfance de la petite Ana, situé dans le présent de la réalisation du film, est raconté par Ana adulte depuis un avenir incertain car les seules images filmées de cet avenir sont des plans fixes de la protagoniste s'adressant à la caméra vingt ans après la réalisation du film - c'est Ana, sous les traits de la jeune Ana Torrent, qui est au centre du niveau narratif second. Cependant, même si la protagoniste apparaît très rarement adulte, il ne semble pas pertinent de qualifier les séquences où elle apparait de flash-forward puisque sa voix off intervient également sur les images de son enfance qu'elle évoque au passé, mais plutôt de flash-back depuis l'avenir. Ce cadre narratif permet de mettre en place un récit entièrement " au passé ", et surtout une narration dans laquelle le présent des spectateurs de l'époque de la sortie du film (1975) est déjà ressenti comme un souvenir. Un passé lui-même hanté par un autre passé, puisque si le récit second commence au moment de la mort du père d'Ana (que l'enfant imagine avoir empoisonné), se poursuit dans les semaines qui suivent pendant les vacances scolaires et s'achève à la rentrée suivante, les réminiscences, les souvenirs et les rêves de la jeune protagoniste évoquent surtout la figure de la mère des trois petites filles, morte plus d'un an auparavant. L'imbrication des différents niveaux d'enchâssement et les métalepses créent un brouillage que le spectateur ne pourra clarifier que peu à peu, par la reconstitution d'un puzzle narratif.

Au sein de cette narration complexe et multiple, la musique joue un rôle fondamental dans le récit filmique et en particulier la chanson Porque te vas interprétée par la jeune chanteuse Jeanette, qui imprègne l'œuvre de ses quatre occurrences. Sortie en 1974, cette chanson était passée relativement inaperçue auprès du public et de la critique lorsque

\footnotetext{
${ }^{1}$ Dans sa thèse publiée en 1987, Claudia Gorbman définit les critères du classicisme musical hollywoodien : invisibilité de l'appareillage de production de la musique; musique conçue pour susciter une écoute inconsciente; la musique traduit les émotions et ponctue la narration; elle constitue un facteur de continuité et d'unité. Gorbman, Claudia, Unheard melodies, Narrative film music, Indiana, Indiana University Press, 1987. Soulignons que, comme tout modèle, celui-ci s'applique à une majorité de film, soumis à ces codes dominants. Si, dans ses premières œuvres, Carlos Saura a également utilisé la musique dans ses fonctions traditionnelles hollywoodiennes, il s'en est par la suite écarté. Cette évolution correspond à un mouvement général de rejet du modèle, présent chez de nombreux réalisateurs dans les années 60, tels Robert Bresson, Eric Rohmer, Jacques Rivette, Ingmar Bergman ou encore Luis Buñuel.

${ }^{2}$ Cette très célèbre Copla, enregistrée par Imperio Argentina en 1935, très populaire pendant la période de la république, a été censurée sous le régime franquiste (et donc très peu chantée) car certaines parties du refrain étaient considérées comme beaucoup trop sensuelles.
} 
Carlos Saura la choisit, en 1975, pour son film Cría cuervos. C'est sa citation dans l'œuvre cinématographique qui est à l'origine de son immense succès international. En effet, à l'occasion du lancement du film, en 1976, le disque est réédité et la chanson fera le tour du monde, sera traduite dans plusieurs langues (y compris le japonais) et sera interprétée par de nombreux chanteurs, jusqu'à aujourd'hui, trente-cinq ans plus tard. Jeanette, en revanche, ne connaîtra plus de très grands succès après ce raz-de-marée médiatique.

Dans le film, la chanson intervient à trois reprises en modalité intradiégétique et une fois en modalité extradiégétique. Seule la dernière occurrence correspond intégralement à la version du disque, deux diffusions sont tronquées et la troisième est manipulée puisque le refrain est répété quatre fois après les deux premiers couplets.

Cette mélodie, elle-même invisible par essence, remplit de multiples fonctions narratives et esthétiques dans son rapport à l'image mouvante et permet de mettre au jour différentes strates invisibles du récit filmique. Elle révèle tout d'abord l'intériorité du personnage d'Ana qui est incapable d'exprimer la douleur de la perte de sa mère adorée et sans doute même d'en être consciente dans un processus de refoulement infantile. Par ailleurs, les différentes citations de la chanson marquent le tissu filmique de leurs occurrences et permettent de mettre en place un récit second constitué par les séquences au sein desquelles elle est diffusée. Enfin, Porque te vas permet de dévoiler le point de vue dissimulé de l'énonciateur filmique que laisse apparaître sa toute dernière citation associée au générique de fin.

\section{Une chanson simple mais ambivalente}

La structure très simple de la chanson s'organise autour d'un couplet reproduisant deux fois la même mélodie et d'un refrain qui intervient entre les deux couplets et se répète à deux reprises à la fin. Le «je » poétique et musical évoque le départ de l'être aimé et les paroles insistent sur l'écoulement du temps (« en mi reloj todas las horas vi pasar»), un temps qui semble ralentir jusqu'à atteindre une sorte d'immobilisme dans une attente sans espoir de retour («junto a las manillas de un reloj / esperarán / todas las horas que quedaron por vivir / esperarán »).

Le noyau signifiant minimal "porque te vas» est extrêmement prégnant car il intervient à plusieurs reprises à la fois dans le premier couplet et dans le refrain. Son sens, pourtant apparemment sans équivoque est rendu ambigu par l'association du texte et de la musique. En effet, «porque te vas » signifie " parce que tu pars », explication de la tristesse de la jeune fille dont le fiancé s'en va. Néanmoins, ces deux termes se distinguent grâce à la différence d'accentuation entre por qué - accentuation tonique de qué - et porque - mot atone. Ici, la musique crée un accent tonique puisque le terme " porque » commence sur une syncope. La syllabe "por» est placée sur la deuxième partie du troisième temps de la mesure à quatre temps et "que» sur le quatrième temps. Le "porque » devient donc naturellement "por qué », transformant une affirmation - parce que tu t'en vas - en interrogation lancinante - pourquoi t'en vas-tu? - démultipliant ainsi la signification de la chanson. Cet aspect est accentué par la répétition incessante du segment " porque te vas », qui tend à l'autonomiser et à renforcer l'interprétation interrogative. De plus, le tout dernier "porque te vas » du refrain est construit sur une ligne mélodique ascendante du la grave au sol moyen, ce qui contribue à renforcer cet aspect interrogatif par l'introduction d'une «intonation musicale». La voix enfantine de Jeanette et son interprétation assez peu 
expressive confèrent également un caractère ambigu à la mélodie, entre détachement de l'interprétation et sens des paroles. Les paroles contrastent aussi avec la rythmique pop et les cuivres qui ponctuent la mélodie. Celle-ci, en sol mineur avec des modulations en si bémol majeur mais harmonisée principalement avec des accords mineurs, est assez mélancolique et s'oppose aussi au caractère entrainant de la rythmique et des cuivres. Tous ces aspects contrastés contribuent à la mise en place d'un équilibre fragile entre gaité et mélancolie, sentimentalisme et distance, évidence et ambigüité, dévoilant une richesse, insoupçonnée à première vue, qui a sans nul doute participé au succès de la chanson.

\section{Porque te vas et l'intériorité d'Ana}

Les deux premières occurrences se succèdent à quelques minutes d'intervalle. Ana, dans la salle de jeu en compagnie de ses sœurs, sort le disque d'un placard, le place sur son petit électrophone et le met en marche (TC: 29mn 50s). Les premières mesures de l'introduction résonnent, puis la fillette articule le début des paroles en même temps que s'élève la voix de la chanteuse. Arrive alors la tante Paulina, la sœur de la mère des orphelines qui se charge de leur éducation depuis la mort de leur père. Paulina enjoint Ana de baisser le son. La petite fille prend un air contrarié et arrête le disque.

La deuxième occurrence intervient quelques secondes après que l'arrivée intempestive de la tante Paulina a interrompu la première citation. Ana enclenche à nouveau le quarante-cinq tours (TC: $31 \mathrm{mn} 21 \mathrm{~s}$ ) et la chanson est diffusée à une forte intensité. À l'exception de la voix de l'aînée, Irène, qui propose à la benjamine Maite de danser avec elle ("¿bailas?»), la musique occupe alors l’intégralité du canal sonore jusqu'à l'interruption de la danse par la sonnerie insistante de la grand-mère qui appelle à l'étage inférieur.

Le deuxième long plan, d'une durée de dix-huit secondes, suit le regard d'Ana qui observe ses deux sœurs en train de commencer à danser. Par des panoramiques fluides, la caméra accompagne les mouvements, malhabiles mais charmants, des petites filles, qui s'approchent et s'éloignent l'une de l'autre. Dès le plan suivant, Ana se joint à ses sœurs et la séquence se poursuit alors que la caméra continue à cadrer successivement les trois enfants qui évoluent gracieusement. Le charme de la scène ${ }^{3}$ des sœurs en train de danser crée une parenthèse d'insouciance au sein de la gravité et de la tension du récit principal. ${ }^{4}$ Une parenthèse que renforce la monopolisation par la musique de tout le canal sonore et dans laquelle Saura se plaît à montrer les évolutions gauches et attendrissantes des trois fillettes.

\footnotetext{
${ }^{3}$ Il s'agit bien d'une scène au sens que lui donne Genette. La danse suivant le déroulement de la chanson en modalité d'écran, durée du récit et durée de l'histoire coïncident et aucune ellipse n'est possible. GENETTE, Gérard, Nouveau discours du récit, Paris, Éditions du Seuil, 1983.

4 Selon Pascale Thibaudeau : «Le moment de complicité que favorise la danse entre les fillettes exprime une forme de résistance à l'ordre que veut imposer Paulina, et qui, bien sûr, renvoie à l'ordre franquiste. » ThiBaudeau, Pascale, Danse et cinéma. L’hybridation des formes dans les films de Carlos Saura, Ouvrage inédit, 2010, p. 44.
} 


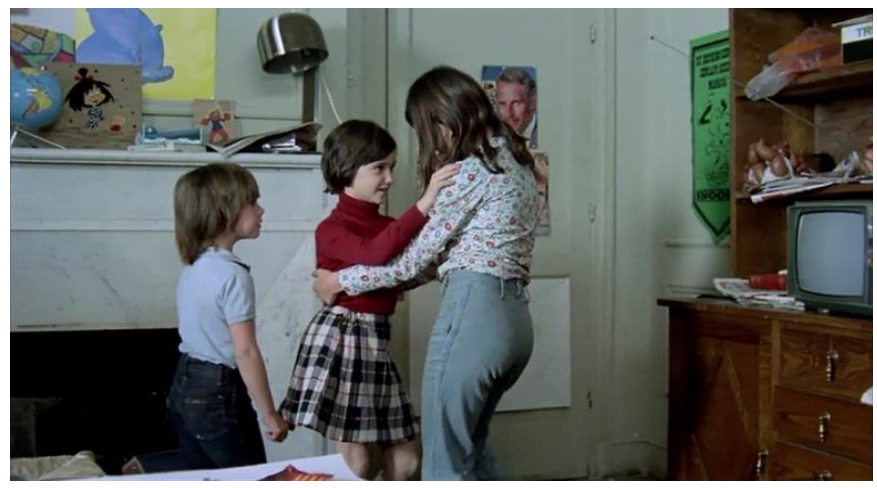

L'association du personnage d'Ana et de la chanson dès ces premières occurrences et la focalisation interne établie au début de la scène par le plan subjectif, mettent en relation la mélodie et la situation d'Ana et permettent de révéler son intériorité. Ce processus est renforcé par l'association naturelle entre la voix enfantine de Jeanette, et le corps de la petite fille, la voix de l'interprète se prêtant naturellement à l'expression de la douleur indicible et invisible d'Ana.

La fillette souffre (« el corazón se pone triste contemplando la ciudad» / «Mon cœur devient triste en regardant la ville.») de l'absence de sa mère ("porque te vas» / «parce que tu t'en vas») tout en ne comprenant pas cette absence ("¿por qué te vas?» / «Pourquoi t'en vas-tu?»). Alors que le temps semble interminable dans cette immense maison triste depuis la disparition de la mère aimée et complice («todas las horas que quedaron por vivir / esperarán» / «Toutes les heures qui n’ont pas été vécues / attendront»). Le dispositif mis en place par la situation d'énonciation, le yo poétique et musical s'exprimant par la voix de Jeanette qui s'adresse à un tú destinataire, facilite dans un premier temps l'association du yo à la fillette et du tú à sa mère.

Dans un deuxième temps, l'identification du spectateur au personnage est également enrichie et consolidée par la subtile mise en place d'un phénomène qui diffère sensiblement de ce qui est défini habituellement comme la fonction empathique de la musique au cinéma, qui selon Michel Chion est créée «[...] par une musique qui est ou semble en harmonie avec le climat de la scène : dramatique, tragique, mélancolique, etc. ${ }^{5}$ La musique vocale possède le pouvoir de traduire subtilement ce que ressent le personnage dans une dimension plurivoque. Si cette séquence était accompagnée, non pas de Porque te vas mais d'une musique instrumentale mélancolique ou nostalgique, le spectateur comprendrait sans doute, grâce au contexte filmique associé à la mélodie, que la fillette est triste et imaginerait que ce sentiment est provoqué par la perte de sa mère qui lui manque. Le lien entre le spectateur et la protagoniste serait alors plutôt de nature sympathique ou encore relèverait de la contagion émotionnelle ${ }^{6}$ lorsqu'il percevrait la tristesse exprimée par la musique. En revanche, l'empathie révèle une capacité à se mettre à la place de l'autre afin de ressentir ou de comprendre ce qu'il éprouve. ${ }^{7}$ L'utilisation de la chanson à la rythmique entraînante

\footnotetext{
${ }^{5}$ CHION, Michel, Un art sonore le cinéma, Paris, Fayard, 1995, p.421.

${ }^{6}$ Selon Elisabeth Pacherie : «La contagion émotionnelle désigne le phénomène de propagation de l'émotion d'un individu à d'autres. C'est un phénomène bien connu de la psychologie des foules et qui se rencontre également chez les bébés, qui répondent aux pleurs d'un autre bébé en commençant eux-mêmes à pleurer. » PACHERIE, Elisabeth, «L'empathie et ses degrés » in BERTHOZ, Alain, JORLAND, Gérard (dir.), L'empathie, Paris, Odile Jacob, 2004, p. 149.

7 «Le mot empathy apparait en 1904, comme traduction de einfublung créé par Théodor Lipps en 1897 pour qualifier la « capacité de s'identifier à autrui, de ressentir ce qu'il ressent. » CHANGEUX, Jean-Pierre, Du vrai, du beau, du bien, une nouvelle approche neuronale, Paris, Odile Jacob, 2008, p.135.
} 
permet de suggérer au spectateur que la souffrance de la fillette n'est pas complètement consciente, à l'image du texte mélancolique de la chanson, « dissimulé » par un arrangement musical plutôt joyeux. Cette même scène accompagnée d'une mélodie au violon dite « empathique » à la signification beaucoup plus univoque ne permettrait pas de révéler la complexité de la psyché enfantine, insouciante - Ana prend plaisir à danser avec ses sœurs mais également profondément blessée par la situation familiale dramatique. Associée à l'image d'Ana, la chanson permet au spectateur d'éprouver lui-même ce manque et de se mettre à la place de la petite fille dans un rapport véritablement empathique au personnage qui va bien au-delà de la notion classique de musique «empathique » souvent redondante par rapport à l'image, tout en introduisant des nuances subtiles de sens.

Cette utilisation de la chanson, étroitement associée au personnage d'Ana permet donc non seulement d'en révéler une part inconsciente, sa souffrance refoulée mais de la faire ressentir au spectateur. Dans le film, cette douleur n'est d'ailleurs jamais clairement exprimée par la fillette sauf très ponctuellement lorsqu'elle se réveille brusquement au milieu de la nuit en appelant sa mère de façon déchirante (une situation qui trouve également un écho dans les paroles de Porque te vas: Cada noche desperté....) La chanson rend donc perceptible une douleur indicible voire inconsciente qui ne peut être montrée à l'image car elle reste dissimulée derrière l'opacité mystérieuse des yeux noirs de la jeune Ana Torrent.

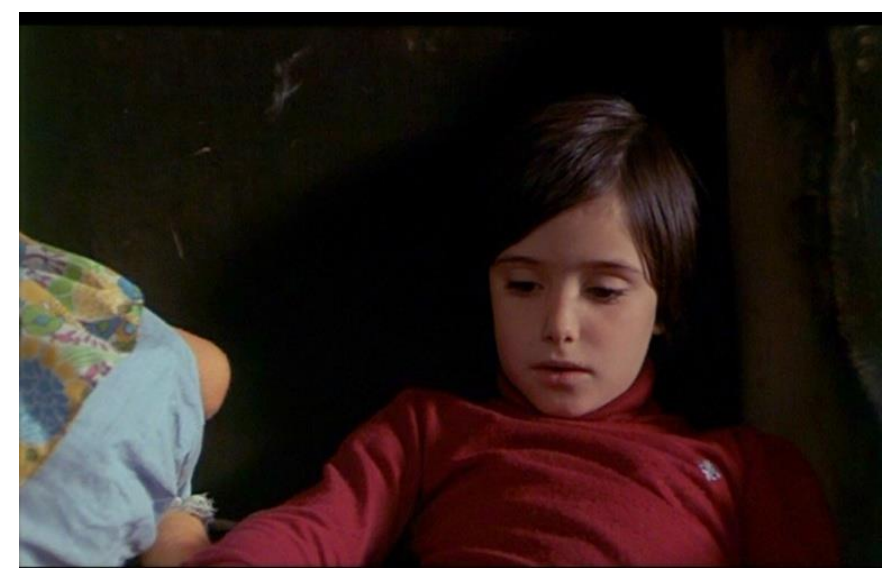

\section{Le parcours narratif}

Outre l'expression des sentiments invisibles d'Ana, les différentes citations de Porque te vas tissent également un réseau signifiant dans le récit filmique car elles relient les différentes séquences pendant lesquelles elles sont diffusée, créant ainsi un véritable parcours «balisé » au sein de la narration filmique, un récit second caché, signalé par la musique : depuis la solitude d'Ana, qui rejette sa tante Paulina, ne pouvant l'accepter en tant que substitut maternel, à une portée symbolique plus large, celle de la souffrance du peuple espagnol écrasé sous la chape du régime franquiste.

Comme nous l'avons évoqué, dès les deux premières citations, un lien s'établit entre la situation de la petite Ana, qui souffre de l'absence de sa mère morte depuis peu et le texte de la chanson. L'arrivée de la tante, qui provoque l'interruption de la chanson lors de la première citation, apparaît comme une intrusion dans l'écoute concentrée d'Ana, murée 
dans le mystère de son monde intérieur. Ces premières occurrences mettent donc en place un réseau de relations invisibles entre, d'une part, la chanson, expression du manque de la mère aimée dont Ana était extrêmement proche, mais également traduction de la solitude, de l'incompréhension et de l'abandon et, d'autre part, le rejet de la tante Paulina qui ne peut, pour Ana, prendre la place de sa mère.

La troisième citation renforce cette opposition puisqu'elle débute à la fin d'une séquence dans laquelle Paulina a eu très peur car Ana s'est présentée avec un pistolet ayant appartenu à son père et l'a pointé sur elle. Paulina a alors violemment giflé Ana, après s'être rendu compte que l'arme était chargée. La chanson débute sur la fin de cette séquence et se poursuit pendant la séquence suivante dans laquelle la fillette, qui écoute à nouveau la mélodie en articulant ses paroles, singe sa tante se maquillant et se recoiffant face à un miroir imaginaire et finit par déclarer tout en regardant la caméra : «Que se muera, quiero que se muera » ("Qu'elle meure, je veux qu'elle meure! »).

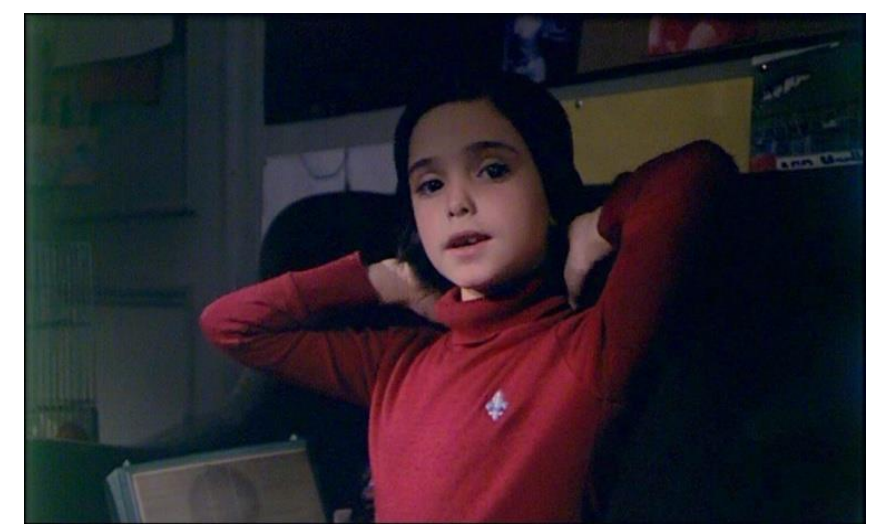

Le début de la séquence suivante, alors que s'achèvent les dernières mesures de la chanson en overlapping ${ }^{8}$ la montre préparant un verre de lait pour sa tante dans lequel elle verse ce qu'elle croit être un poison violent : du bicarbonate de soude. Le manque de la mère se transforme en haine pour Paulina et la solitude n'en est qu'accentuée; la tante est violemment rejetée et sa mort désirée par une invocation magique au miroir imaginaire dont la caméra prend la place. En effet, le tout dernier regard d'Ana est bien adressé à la caméra dans une transgression des règles de la narration classique au cinéma, plaçant la caméra au cœur d'un dispositif magique déclenché par la musique. Ce dispositif fonctionne comme une inversion ironique du conte de Charles Perrault, Blanche-neige, où désormais c'est la belle-fille qui, après s'être contemplée dans le miroir - en jouant néanmoins le rôle de sa tante -, décide de se débarrasser de la marâtre en lui offrant non pas une pomme mais un verre de lait empoisonné.

La seule citation extradiégétique de la chanson clôt le film. Les premières mesures résonnent alors que les trois petites filles, cadrées en plan général en forte plongée,

\footnotetext{
${ }^{8}$ La musique est en overlapping (chevauchement) lorsqu'elle débute au cours d'une séquence et se poursuit pendant la séquence suivante.

${ }^{9}$ Le regard à la caméra est en effet perçu comme un signe d'énonciation qui contribue à dévoiler l'illusion fictionnelle. Selon André Gaudreault: "Au cours de son histoire, le cinéma a forgé des procédures d'effacement ou d'atténuation au point que l'on a pu écrire que "la particularité du texte classique [était] d'occulter complètement l'instance discursive qui le produit, comme s'il n'était que la simple transcription d'une continuité antérieure et homogène" (Marie, 1976: 24), de sorte que, à la lettre, "les événements semblent se raconter eux-mêmes." " GAUDREAUlT, André, JOST, François, Le récit cinématographique, Paris, Nathan, 1990, p. 44.
} 
prennent à pied la direction leur école à travers la ville. Telles des petits points dérisoires au milieu de la foule, elles sont écrasées par la plongée puis séparées de la caméra par le flot des voitures qui barre l'espace et enfin emportées dans le tourbillon des enfants qui se précipitent à l'intérieur de l'école. Les fillettes paraissent perdues dans l'espace immense et fourmillant de la grande ville, rendu menaçant par l'angle de caméra, et dans l'anonymat de la foule. Le temps semble s'arrêter lorsqu'un plan rapproché fixe cadre Ana, puis la multitude des écolières en uniforme qui traverse le champ de gauche à droite. C'est sur un plan général de la ville en plongée que s'égrènent les dernières notes du morceau. L'enfermement et la solitude mis en avant par la chanson se concluent ici par une dernière séquence qui semble souligner le caractère inexorable de la situation des fillettes et d'Ana en particulier. Tout d'abord prisonnières de la grande maison où elles sont soumises à l'autorité de leur tante, elles semblent écrasées par la grande ville, puis enfermées à nouveau à l'école.

La chanson tisse donc des relations complexes entre les différentes séquences qui font écho au sens mis en avant par le rapport entre paroles et musique: sensation d'abandon, incompréhension de la perte de l'être aimé qui se traduit par le rejet de la mère de substitution, temps qui s'écoule inexorablement dans une solitude inchangée et un enfermement sans espoir.

\section{La révélation de l'énonciateur filmique}

Il est également possible de lire dans cette progression narrative, au-delà de la situation douloureuse de la petite Ana, l'expression d'une souffrance collective au sein d'une société bloquée par la persistance d'un régime politique autoritaire pourtant proche de sa fin, puisque le film a été tourné en 1975, année de la mort de Franco. Le passage de la modalité intradiégétique à la modalité extradiégétique, de l'intériorité d'Ana au commentaire extérieur serait une clé de cette lecture symbolique. Celle d'une société, en partie orpheline, qui souffre, comme Ana, qui rejette en silence la tutelle d'une autorité parentale imposée et qui regrette «las horas que quedaron por vivir», les heures qui n'ont pas pu être vécues par une partie d'un peuple réduit au silence depuis de longues années. En effet, la dernière occurrence de la chanson est associée au générique de fin, un de ces espaces de l' «entredeux » où l'énonciation filmique et ses composantes multiples se dévoilent et révèlent au spectateur qu'il sort d'un monde fictionnel façonné de toute pièce. La révélation de cet « envers du décor» associé à la diffusion extradiégétique qui relève également directement de l'énonciation puisqu'aucune cause diégétique ne peut lui être attribuée permet donc d'interpréter cette dernière diffusion comme un message indirect de l'énonciateur filmique qui révélerait ainsi son point de vue. Cette dernière séquence est cependant bien représentative de l'œuvre ouverte de Saura, qui n'est jamais univoque. La plongée écrase effectivement les fillettes qui passent en outre d'un lieu clos à un autre lieu clos. Néanmoins, la puissance de la diffusion de la chanson, mettant en avant la rythmique et les interventions des cuivres assez gais, l'échelle des plans, le vaste espace de la ville et le panoramique final sur Madrid peuvent également évoquer un certain espoir de libération (Malgré tout les fillettes sortent enfin de la maison) à une date clé de l'histoire de l'Espagne.

La musique vocale a donc permis à Saura de révéler l'invisible dans une œuvre qui échappe à toute interprétation étroite de son sens comme d'ailleurs l'ensemble de la 
filmographie de ce grand auteur. Le dévoilement de l'invisible est rendu possible grâce au fonctionnement très spécifique de la chanson et plus particulièrement grâce au processus de resémantisation des paroles. Cette resémantisation intervient à deux niveaux : au niveau de la musique tout d'abord qui les nuance et peut même en modifier la portée, puis à celui du texte filmique qui en oriente également le sens dans un deuxième temps. La douleur indicible de la petite Ana, invisible à l'écran, tout comme celle de toute une partie du peuple espagnol, est alors rendue perceptible par la magie de l'association des paroles, de la musique et de l'image. 